\title{
Real-time latching control of wave energy converter with consideration of wave force prediction
}

\author{
Liang Li \\ Department of Naval Architecture, \\ Ocean and Marine Engineering \\ University of Strathclyde \\ Glasgow, UK \\ liang.li@strath.ac.uk
}

\author{
Yan Gao \\ Department of Naval Architecture, \\ Ocean and Marine Engineering \\ University of Strathclyde \\ Glasgow, UK \\ yan.gao@strath.ac.uk
}

\author{
Zhiming Yuan \\ Department of Naval Architecture, \\ Ocean and Marine Engineering \\ University of Strathclyde \\ Glasgow, UK \\ zhiming.yuan@strath.ac.uk
}

\begin{abstract}
A real-time latching controller with consideration of wave force prediction is developed and applied to a heaving point-absorber to maximize its energy absorption. The control scheme is based on the combination of optimal command theory and first order-one variable grey model $\operatorname{GM}(1,1)$. By forecasting the wave forces in the near future, the control action at the next instant is deduced. Simulation results show that the energy absorption is increased due to the real-time controller. Therefore, the developed real-time controller is applicable to an industrial wave energy converter (WEC) in random waves. The effect of wave force prediction deviation is also examined. It is shown that the control efficiency is reduced in the presence of prediction deviation.
\end{abstract}

Keywords - real-time latching control, wave force prediction, optimal command theory, energy absorption, wave energy converter, renewable energy

\section{INTRODUCTION}

It is expected that the global demand for energy will climb up to 25 percent by 2040 and the world is pursuing economic and renewable energy sources to keep up with this considerable demand growth [1]. Compared with other sustainable energy resources, wave energy is a kind of resource with high power density and all-day availability. Owing to these advantages, wave energy is regarded as a prospective solution to the sustainable generation of power. Various types of wave energy converters have been developed to harvest energy from ocean waves [2-4].

Given that the energy extraction efficiency of WECs is usually relatively (especially in off-resonance state), various control methods were developed to maximize the energy absorption. Babarit et al. [5] studied how the declutching control influenced the energy absorption of a WEC in regular and irregular waves. Merigaud and Ringwood [6] used the pseudo-spectral control to maximize the energy absorption of a one-degree of freedom WEC. Babarit and Clement [7] assessed the benefits produced by the latching control. Based on the pregenerated wave elevations, the optimal command theory was applied to determine the control law. Henriques et al. [8] applied latching control to an oscillating-water-column WEC. The sensitivity of energy absorption to receding horizon length was examined. In spite of the well-developed control strategies, most WEC control assume that the wave forces are already know, implying that they are not applicable in real practice where no one is able to know the wave forces in the future.

Actually, it is necessary to predict the wave forces if a controller is to be implemented to a WEC. Two categories of prediction approaches are available. The first group is the spatial prediction, which forecasts the wave information at a certain point based on the observations at nearby locations. This type of approach can be effectively applied in the WEC array as each device operates as an observation station [9]. The second group forecasts sea waves with the collection of past wave information right at this point, which is by nature a regression method and does not require the dynamic model of the random process. Consequently, it is applicable to the prediction of many variables, such as wave elevation, wave force, floater velocity, etc. Fusco and Ringwood [10] came up with a linear autoregressive model, which implicitly considered the cyclical behaviour of waves. Ge and Kerrigan [11] predicted the wave elevations using autoregressive moving average model. Halliday et al. [12] utilized the fast Fourier transformation to predict the random sea waves. A wave prediction model based on the grey model was developed by Truong and Ahn [13].

A predictive real-time latching controller is developed in this work and applied to a heaving point-absorber to maximise its energy absorption. The developed control scheme is based on the combination of optimal command theory and first orderone variable grey model $\operatorname{GM}(1,1)$. The energy capture performances with and without the predictive real-time controller are investigated to examine whether the proposed control strategy can work effectively in real practice. Besides, the effect of prediction deviation on the control efficiency will be evaluated as well.

\section{MODEL DESSCRIPTION}

A heaving point-absorber as shown in Fig. 1 is considered in this work. The floater is a $5 \mathrm{~m}$ hemisphere, which is rigid connected to the power take-off (PTO) system. The point- 
absorber is only allowed to oscillate up and down. The draft of the floater is $5 \mathrm{~m}$. The PTO system is simplified as a springdamper system. The restoring stiffness $K$ is $78.8 \mathrm{kN} / \mathrm{m}$, and the damping coefficient $C$ is $8.14 \times 10^{5} \mathrm{~kg} / \mathrm{s}$.

Fig. 1. The heaving point-absorber.

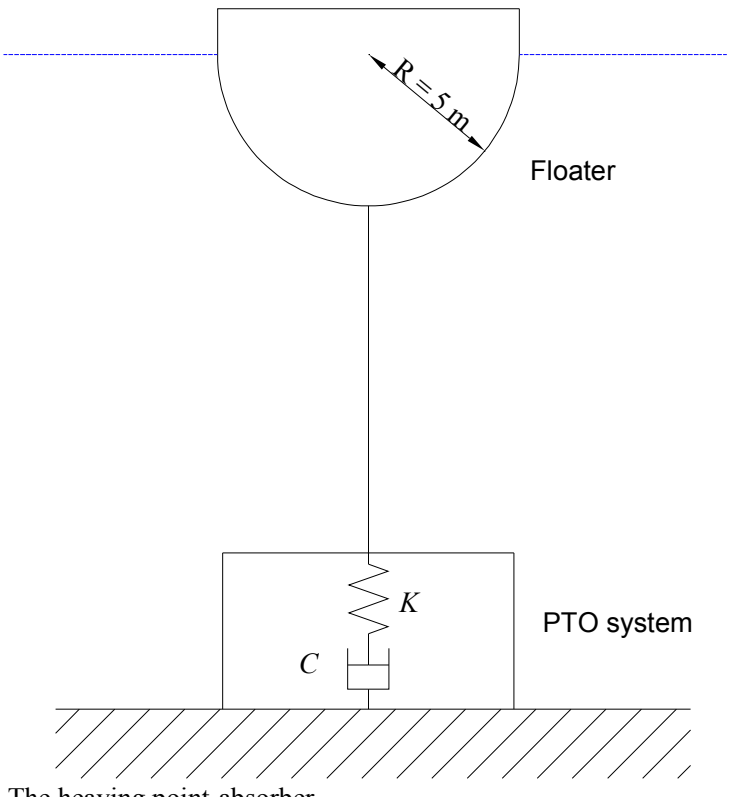

\section{A. Dynamics of the Floater}

Based on the impulse response theory [14], the timedomain motion equation of the floater is given by

$$
\begin{aligned}
& (M+m) \ddot{z}(t)+\int_{0}^{t} H(t-\tau) \dot{z}(\tau) d \tau+\rho g \pi R^{2} z(t) \\
= & F_{\text {wave }}(t)-C \dot{z}(t)-K z(t)-\beta(t) c \dot{z}(t)
\end{aligned}
$$

where $M$ is the mass of the floater and $m$ is the added mass corresponding to infinite frequency. $\mathrm{z}, \dot{z}$, and $\ddot{z}$ are the displacement, velocity, and acceleration vectors. $F_{\text {wave }}$ is the wave excitation force. $\beta(t)$ is the binary control sequence. When $\beta=1$, the floater is locked; when $\beta=0$, it is free to oscillate. The point-absorber switches abruptly between two states $(\beta=0,1)$ so that the latching control is a bang-bang control. $c$ is the damping coefficient representing the damping force used to lock the floater. $H$ is the so-called retardation kernel function which represents the memory effect of radiation force. It can be obtained from either the added mass $a(\omega)$ or the potential damping $b(\omega)$

$$
H(t)=\frac{2}{\pi} \int_{0}^{\infty} \frac{b(\omega)}{\omega} \sin (\omega t) d \omega=\frac{2}{\pi} \int_{0}^{\infty} a(\omega) \cos (\omega t) d \omega
$$

where $\omega$ is the wave oscillation frequency. Eq. (1) is the classical time-domain motion equation used in seakeeping problem. It is inconvenient to implement the control strategy using Eq. (1). Therefore, an alternative model is developed to calculate the motions of the point-absorber in random waves, in which the convolution term is replaced by a state-space representation.

$$
\begin{aligned}
& \int_{0}^{t} H(t-\tau) \dot{z}(\tau) d \tau=\vec{C} u(t) \\
& \dot{u}(t)=\vec{A} u(t)+\vec{B} \dot{z}(t)
\end{aligned}
$$

Where $\vec{A}, \vec{B}$, and $\vec{C}$ are constant matrices identifying the system, which can be deduced from the hydrodynamic coefficients of the point-absorber. The calculation of these matrices is knowns as system identification. Define a state vector $x=[z, \dot{z}, u]^{\mathrm{T}}$ with dimension $(n+2) \times 1$. Then (1) is reexpressed as

$$
\begin{aligned}
\dot{x} & =\gamma \cdot x+\eta \\
\gamma & =\left[\begin{array}{ccc}
0 & 1 & 0 \\
-\frac{\rho g \pi R^{2}+K}{M+m} & -\frac{C+\beta c}{M+m} & -\frac{\vec{C}}{M+m} \\
\overrightarrow{0} & \vec{B} & \vec{A}
\end{array}\right] \\
\eta & =\left[\begin{array}{c}
0 \\
\frac{F_{\text {wave }}}{M+m} \\
\overrightarrow{0}
\end{array}\right]
\end{aligned}
$$

Eq. (4) is a first-order, one-variable differential formula, which is very easy to handle. Given the initial condition $x(0)=$ 0 , it becomes a classical initial-value problem and time series of floater movement can be obtained by the 4th Runge-Kutta method. Then, the average energy absorption during simulation interval $[0, T]$ is given by

$$
P=\int_{0}^{T} C \cdot \dot{z}(t, \beta)^{2} d t / T
$$

\section{REAL-TIME LATCHING CONTROL}

\section{A. Optimal Command Theory}

Assuming that the wave forces during an interval $\left[t_{1}, t_{2}\right]$ are already known, our objective is to maximise the average energy absorption through the binary control sequence $\beta(t)$. From a mathematical point of view, it is required to find the maximum of $P$ subject to constraint Eq. (4). Now, define a Hamiltonian $H$

$$
H=C \dot{z}^{2}+\lambda \cdot(\gamma x+\eta)
$$

According to the Pontryagin maximum principle, the optimal $\beta$ is the one maximising the Hamiltonian at every time step throughout $\left[t_{1}, t_{2}\right]$. Apparently, the Hamiltonian reaches the maximum value on condition that

$$
\beta=\left\{\begin{array}{cc}
1 & \lambda_{2} c \dot{z}<0 \\
0 & \text { otherwise }
\end{array}\right.
$$

Eq. (7) is the criteria to judge whether the point-absorber should be locked or not. At this point, the next work is to calculate $\lambda$ and $\dot{z}$. Please note that $\lambda$ follows the following relationship. 


$$
\begin{aligned}
& \dot{\lambda}_{i}=-\frac{\partial H}{\partial x_{i}}(t, x, \beta), i=1,2, . ., n \\
& \lambda\left(t_{2}\right)=\overrightarrow{0}
\end{aligned}
$$

Eq. (8) cannot be solved numerically like an initial value problem as the final condition is given here. An iterative process is applied here. Firstly, run the simulation with $\beta(t)=0$ to obtain the motions free of latching action. Subsequently, determine $\lambda$ by integrating (8) backwards from $t=t_{2}$ to $t=t_{1}$ Then, the control sequence $\beta(t)$ is deduced. Iterating the process with the determined control sequence until the results converge, the optimal control sequence $\beta(t)$ can be derived.

\section{B. Wave Force Prediction}

The application of optimal command theory is based on the assumption that the wave forces are already known. Therefore, it is required to predict the wave forces before applying the optimal command theory to deduce control sequence. The first order-one variable grey model $\operatorname{GM}(1,1)$ is used here to predict the wave force. In the grey prediction theory, the prediction is based on the collection of historical raw data without requirement of the mathematical model of the dynamic process. At time step $t_{i}$, start the forecasting process by collecting at least 4 consecutive raw data $X$ over the past few seconds. Moreover, the raw data must be non-negative. To meet the requirement, a positive offset $Q=\left|2 \cdot \min \left(x_{i}\right)\right|$ is added to the raw data so that the data will be positive. The offset $Q$ is subsequently deducted from the predicted results at the end of the forecasting process.

$$
X=\left(x_{1}, x_{2}, \ldots, x_{n}\right)+Q, n \geq 4
$$

Obtain an accumulated series $Y$ from $X$

$$
\begin{aligned}
& Y=\left(y_{1}, y_{2}, \ldots, y_{n}\right) \\
& y_{k}=\sum_{i=1}^{k} x_{i}, k=1,2, \ldots, n
\end{aligned}
$$

Create the so-called background series $Z$

$$
\begin{aligned}
& Z=\left(z_{2}, z_{3}, \ldots, z_{n}\right) \\
& z_{k}=\left(y_{k}+y_{k-1}\right) / 2
\end{aligned}
$$

Set the grey differential formula

$$
x_{k}+a z_{k}=b, k=2,3, . ., n
$$

and acquire parameters $a$ and $b$ with the least square method

$$
\begin{aligned}
& {\left[\begin{array}{l}
a \\
b
\end{array}\right]=\left(A^{T} A\right)^{-1} A^{T} B} \\
& A=\left[\begin{array}{cc}
-z_{2} & 1 \\
-z_{3} & 1 \\
\vdots & \vdots \\
-z_{n} & 1
\end{array}\right], B=\left[\begin{array}{c}
x_{2} \\
x_{3} \\
\vdots \\
x_{n}
\end{array}\right]
\end{aligned}
$$

Establish the first order-one variable grey model $\operatorname{GM}(1,1)$ to predict the random signal within interval $\left[t_{i+1}, t_{i+p}\right]$

$$
\begin{aligned}
& \hat{x}_{n+p}=\hat{y}_{n+p}-\hat{y}_{n+p-1}-Q \\
& \hat{y}_{n+p}=\left(y_{1}-\frac{b}{a}\right) e^{-a(n+p-1)}+\frac{b}{a}
\end{aligned}
$$

Where $\hat{x}_{n+p}$ is the predicted data at time step $t_{i+p}$. At time step $t_{i}$, collect raw data and predict the data over $\left[t_{i+1}, t_{i+1}+p \cdot d t\right]$. Repeat the forecasting process at step $t_{i+1}$ again to forecast the data over $\left[t_{i+2}, t_{i+2}+p \cdot d t\right]$. By collecting raw data and predicting future data alternately, the grey model can provide real-time prediction of the random signal.

\section{Predictive Real-time Control}

Based on the combination of $\operatorname{GM}(1,1)$ model and optimal command theory, a predictive real-time controller is developed. Fig. 2 illustrates the procedure of the real-time control strategy. At time step $t_{i}$, predict the wave forces over a certain receding horizon $\left[t_{i+1}, t_{i+1}+\Delta t\right]$ using the $\operatorname{GM}(1,1)$ model. Since the wave forces have been predicted, one can use the optimal command theory to deduce the optimal control sequence over $\left[t_{i+1}\right.$, $\left.t_{i+1}+\Delta t\right]$. At time step $t_{i+1}$, apply the control action which has been predicted at the previous step and repeat the process again to predict the control action at time step $t_{i+2}$. Please note that only the predicted control sequence $\beta\left(t_{i+1}\right)$ is adopted. By receding forward this algorithm, the real-time control is implemented throughout the entire interval. Such a control strategy is also known the receding horizon control or the model predictive control.

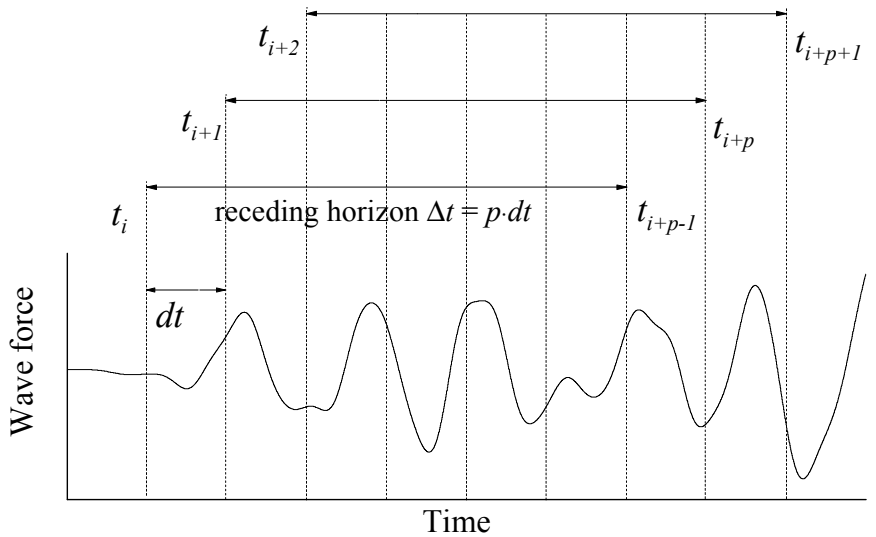

Fig. 2. Receding horizon control.

\section{VALIDATION}

Firstly, we validate the numerical model in the absence of the latching control. The energy absorption in a set of unit regular waves with various oscillation frequencies are simulated. The results are compared with those suggested by frequency-domain hydrodynamic analysis programme Wadam [15], developed by DNV-GL. Please note that the pointabsorber is a linear system without the latching control so that Wadam is applicable here. The PTO system force is modelled with the 'additional damping' and 'additional stiffness' options provided in Wadam. As displayed in Fig. 3, the agreement between the two simulation tools are very good. 


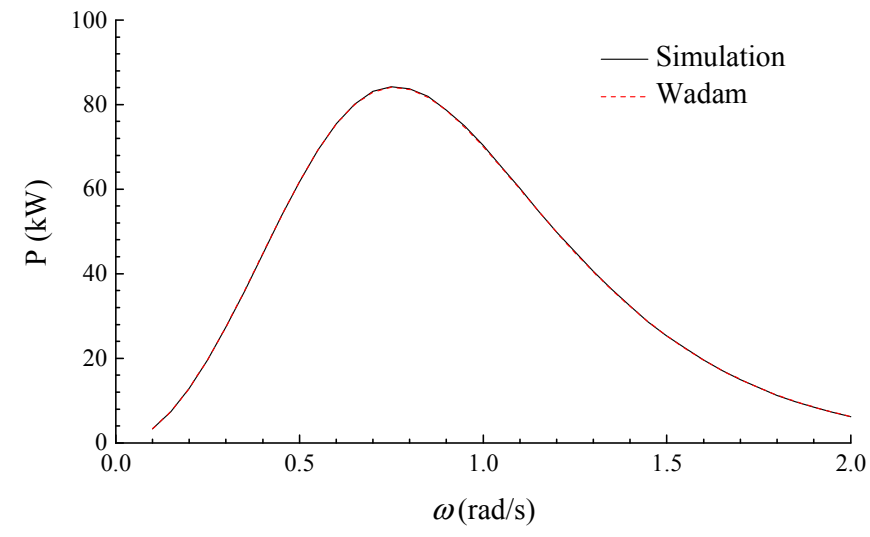

Fig. 3. Energy absorption in regular waves.

\section{RESULTS AND DISCUSSIONS}

\section{A. Regular Waves}

Fig. 4 plots the energy absorbed by the WEC in regular waves. The wave amplitude is $1 \mathrm{~m}$. The receding horizon is set to $0.2 T$ ( $T$ is the period of the regular wave). It is desirable to see that the energy extraction is improved with the real-time controller. It proves that the real-time controller can work effectively in real practice. Fig. 5 illustrates the effect of latching action on the dynamics of the WEC. As expected, the response of the WEC is a succession of locked and ramp stages. The floater is latched for a certain duration when the velocity vanishes. Once released, the velocity starts to climb up rapidly. Due to the latching action, the amplitude of the velocity is augmented. At the same time, the velocity phase is tuned (see Fig. 6). With the regulation of the real-time controller, the velocity is generally in phase with the wave force. This property has been widely used as the criterion to calculate latching control since the work of Budal and Falnes [16]. Therefore, Fig. 5 can be used to validate the developed controller.

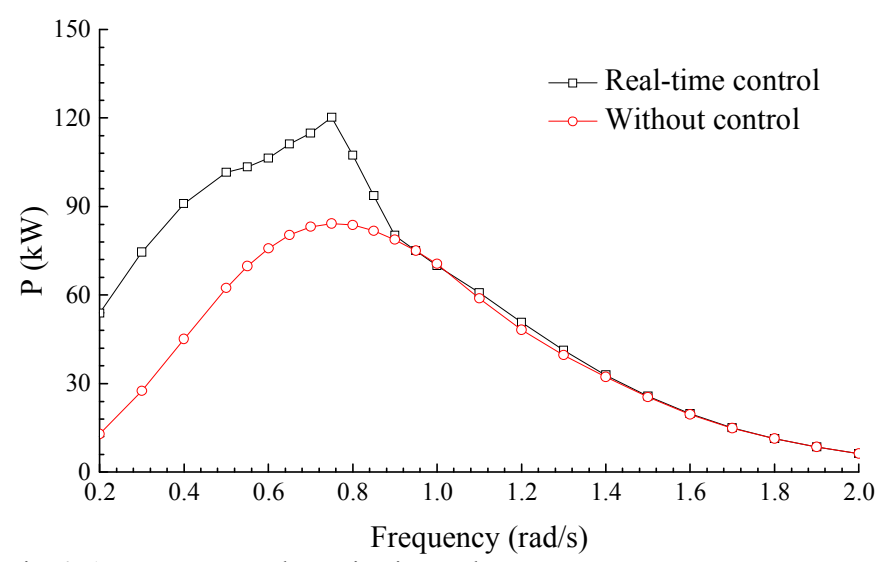

Fig. 4. Average energy absorption in regular waves.

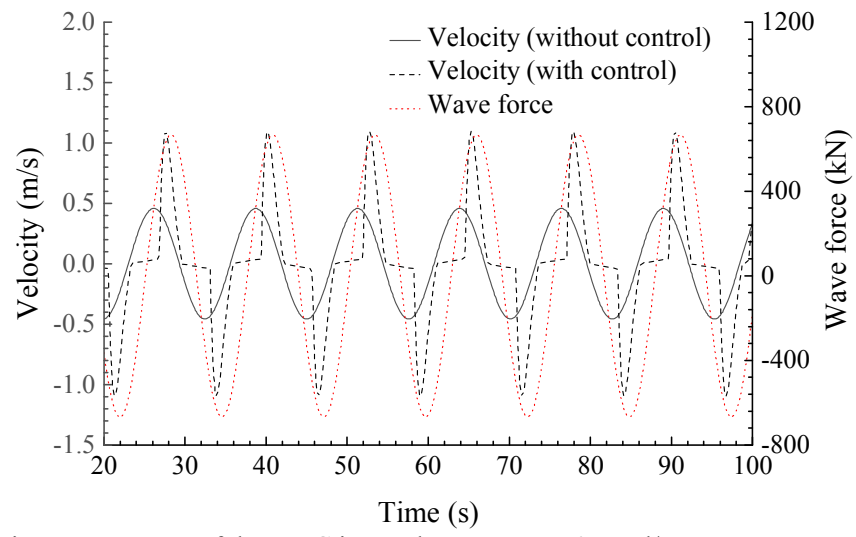

Fig. 5. Responses of the WEC in regular wave, $\omega=0.5 \mathrm{rad} / \mathrm{s}$.

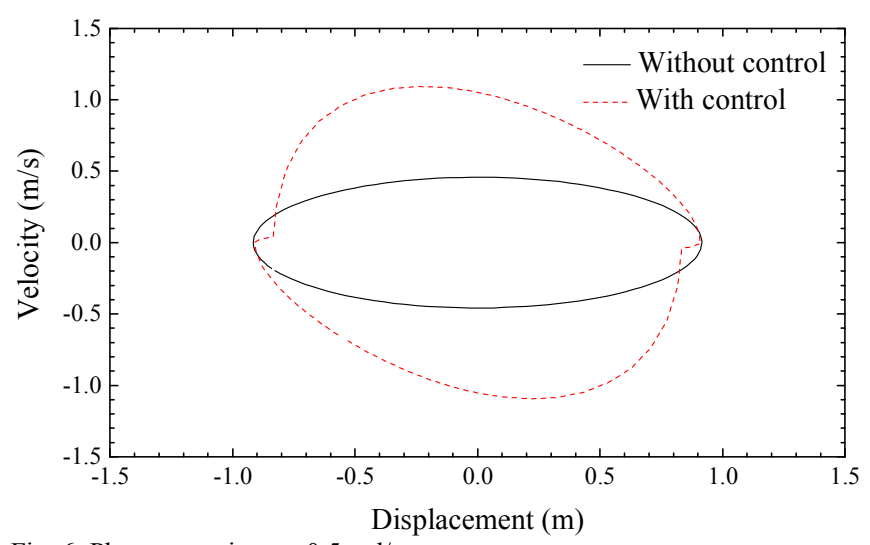

Fig. 6. Phase portrait, $\omega=0.5 \mathrm{rad} / \mathrm{s}$.

Nevertheless, the real-time controller seems to be effective only within low frequency range. When the point-absorber is subject to high frequency waves, the controller no longer helps. As pointed out in [17], the duration of a single ramp is roughly equal to half resonant period of the WEC on condition that the PTO damping coefficient $C$ is sufficiently small (weak damping). Therefore, the solution of control sequence is only available when the wave period is larger than the resonant period of the WEC. Although the damping coefficient $C$ adopted is very high to harvest more wave energy, this property can still help to interpret why the real-time control is effective in low frequency regular waves.

\section{B. Irregular Waves}

\section{TABLE I. WAVE CONDITIONS}

\begin{tabular}{cccc}
\hline & Case 1 & Case 2 & Case 3 \\
\hline $\mathrm{H}_{\mathrm{s}}(\mathrm{m})$ & 2 & 4 & 6 \\
$\mathrm{~T}_{\mathrm{p}}(\mathrm{s})$ & 11.11 & 12.33 & 13.27 \\
\hline
\end{tabular}

Three random wave conditions are considered to examine the effectiveness of the predictive real-time controller (see TABLE I). The receding horizon is set to $2 \mathrm{~s}$. TABLE II lists the average energy absorption in the three wave conditions concerned. Owing to the control regulation, the average energy absorption is increased by over $40 \%$. It proves that the proposed real-time control scheme is effective in the real world as well. Consequently, it can be implemented to an industrial WEC product directly. 
TABLE II. AVERAGE ENERYG ABSORPTION

\begin{tabular}{lll}
\hline & Without control & With control \\
\hline Case 1 & $34.8 \mathrm{~kW}$ & $49.9 \mathrm{~kW}$ \\
Case 2 & $140.0 \mathrm{~kW}$ & $201.1 \mathrm{~kW}$ \\
Case 3 & $284.0 \mathrm{~kW}$ & $428.8 \mathrm{~kW}$ \\
\hline
\end{tabular}
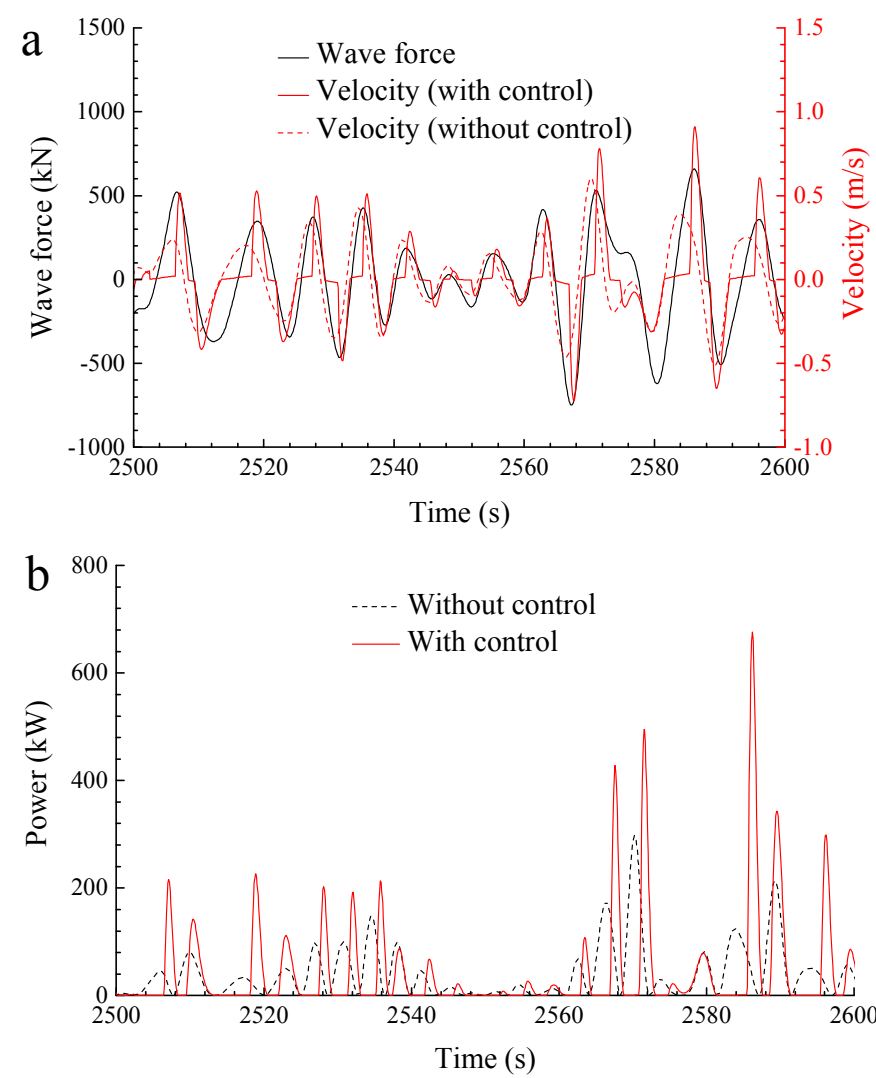

Fig. 7. Responses of the point-absorber, Case1. (a) velocity and wave force; (b) energy absorption.

Fig. 7 compares the responses of the WEC with and without the real-time latching control in irregular waves. From the red solid curve representing velocity with the application of latching control, it is obvious that the floater is locked randomly. At the same time, the amplitude of velocity is amplified under the action of latching control. Moreover, one can see that the velocity phase is tuned. The velocity is generally in phase with the wave force under the action of latching control. The effect of real-time latching control on the energy harvesting is illustrated in Fig. 7 (b), where the power output peak is increased significantly representing the benefit produced by the real-time control. Nevertheless, the power output becomes less stable as the PTO system is locked occasionally. The potential damage of this property to the integrity of PTO mechanical facilities deserves further investigation.

\section{Effect of Prediction Deviation}

Since the wave forces over receding horizon $\left[t_{i+1}, t_{i+1}+\Delta t\right]$ is forecasted, the predicted wave forces differ from the real ones. Such prediction deviation may have an influence on the realtime controller performance.
Fig. 8 displays the prediction deviation effect on the energy absorption. As shown, the point-absorber extracts more wave power with the real-time controller when the prediction deviation is omitted, implying that the prediction deviation has a negative effect on the efficiency of the real-time controller.

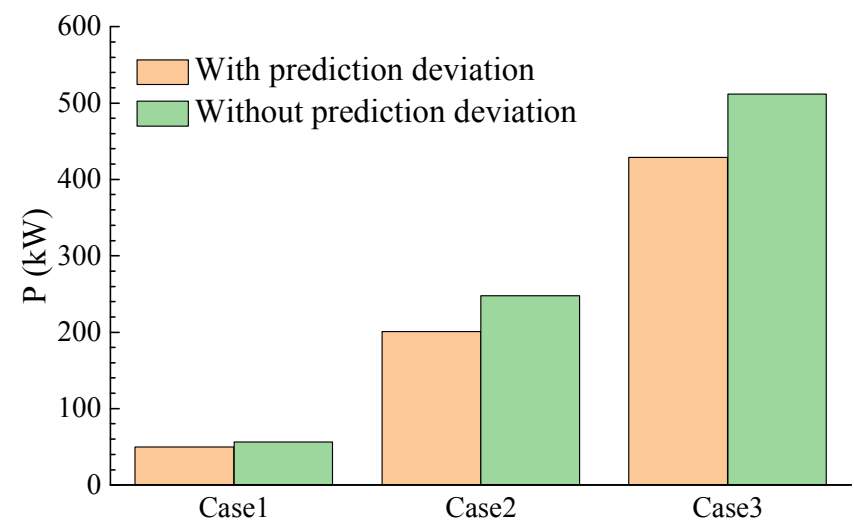

Fig. 8. Effect of prediction deviation on the control efficiency.

Since the point-absorber is subject to the real wave forces no matter whether the wave forces over $\left[t_{i+1}, t_{i+1}+\Delta t\right]$ is predicted or not. The discrepancies in Fig. 8 is completely caused by the control law $\beta$, which is deduced on the forecasted wave forces rather than the actual ones. Fig. 9 plots the control law deduced by the real-time controller with and without prediction deviation. In the presence of prediction deviation, the control law is somewhat varied compared with that free of deviation. It indicates that the controller sometimes makes the wrong decision regarding whether locking the pointabsorber or not. For example, the point-absorber should be released at $t=0.8 \mathrm{~s}$ whereas it is locked due to the prediction deviation. Consequently, the controller efficiency is reduced.

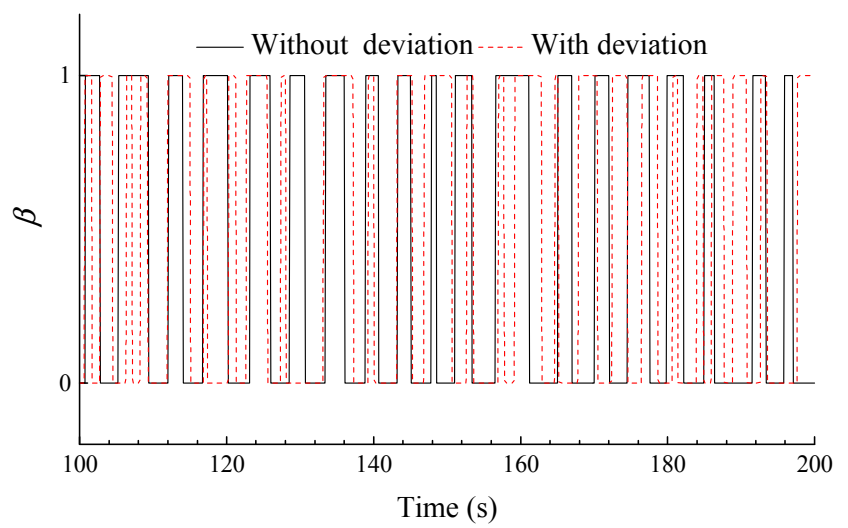

Fig. 9. Control law with and without prediction deviation, Case1.

\section{CONCLUSIONS}

Considering that most previous WEC control studies assume that the wave forces are already known and are not applicable to a true wave energy device, a predictive real-time controller is developed in this study. The real-time control scheme is based on the combination of optimal command theory and grey model $\operatorname{GM}(1,1)$. The wave forces over the prediction interval are forecasted with the grey model $\operatorname{GM}(1,1)$ and then the control sequence is deduced by using the optimal 
command theory. By receding the control scheme forward at each time step, the real-time control is implemented.

The movement of the point-absorber is a succession of latched and ramp stages in regular waves in the presence of latching action. By regulating the velocity and making it in phase with the wave excitation forces, the real-time controller enlarges the energy absorption. For the random wave conditions considered in this study, the energy absorption is increased by more than $40 \%$ with application of the real-time controller. Simulation results manifest that the real-time control strategy developed in this paper can work efficiently in real world, where the wave forces are stochastic and unknown. Besides, the prediction deviation has a negative effect on the efficiency of the real-time controller.

\section{REFERENCES}

[1] ExxonMobile, "Outlook for Energy: A View to 2040", 2017.

[2] L. Li, Y. Gao, Z.M. Yuan, S. Day, Z.Q. Hu, "Dynamic response and power production of a floating integrated wind, wave and tidal energy system", Renew Energ 116 (2018) 412-422.

[3] F. He, Z.H. Huang, A.W.K. Law, "An experimental study of a floating breakwater with asymmetric pneumatic chambers for wave energy extraction", Appl Energ 106 (2013) 222-231.

[4] A.F.O. Falcao, J.C.C. Henriques, "Oscillating-water-column wave energy converters and air turbines: A review", Renew Energ 85 (2016) 1391-1424.

[5] A. Babarit, M. Guglielmi, A.H. Clement, "Declutching control of a wave energy converter", Ocean Eng 36(12-13) (2009) 1015-1024.
[6] A.P.L. Merigaud, J.V. Ringwood, "Improving the computational performance of non-linear pseudo-spectral control of wave energy converters", IEEE T Sustain Energ PP(99) (2017) 1-1.

[7] A. Babarit, A.H. Clement, "Optimal latching control of a wave energy device in regular and irregular waves", Appl. Ocean Res 28(2) (2006) 77-91.

[8] J.C.C. Henriques, L.M.C. Gato, A.F.O. Falcao, E. Robles, F.X. Fay, "Latching control of a floating oscillating-water-column wave energy converter", Renew Energ 90 (2016) 229-241.

[9] C.P. Tsai, C. Lin, J.N. Shen, "Neural network for wave forecasting among multi-stations", Ocean Eng 29(13) (2002) 1683-1695.

[10] F. Fusco, J.V. Ringwood, "Short-Term Wave Forecasting for Real-Time Control of Wave Energy Converters", IEEE T Sustain Energ 1(2) (2010) 99-106.

[11] M. Ge, E.C. Kerrigan, "Short-term ocean wave forecasting using an autoregressive moving average model", Control (CONTROL), 2016 UKACC 11th International Conference on, IEEE, 2016, pp. 1-6.

[12] J.R. Halliday, D.G. Dorrell, A.R. Wood, "An application of the Fast Fourier Transform to the short-term prediction of sea wave behaviour", Renew Energ 36(6) (2011) 1685-1692.

[13] D.Q. Truong, K.K. Ahn, "Wave prediction based on a modified grey model $\operatorname{MGM}(1,1)$ for real-time control of wave energy converters in irregular waves", Renew Energ 43 (2012) 242-255.

[14] W. Cummins, "The impulse response function and ship motions", David Taylor Model Basin, Washington DC, 1962, pp. 101-109.

[15] D.N. Veritas, "WADAM-Wave Analysis by Diffraction and Morison Theory, SESAM user's manual”, Høvik, 1994.

[16] K. Budal, J. Falnes, "Interacting point absorbers with controlled motion, in Power from Sea Waves", BM Count, Academic Press 1980.

[17] A. Babarit, G. Duclos, A.H. Clement, "Comparison of latching control strategies for a heaving wave energy device in random sea", Appl. Ocean Res 26(5) (2004) 227-238. 